\title{
Current Treatments for Anxiety and Obsessive-Compulsive Disorders
}

\author{
Sehrish Sayed, $M P H^{1}$ \\ Sarah R. Horn, $B A^{1}$ \\ James W. Murrough, $M D^{1 *, 2,3}$
}

\author{
Address \\ ${ }^{1 *}$ Mood and Anxiety Disorders Program, Department of Psychiatry, Icahn \\ School of Medicine at Mount Sinai, 1 Gustave L. Levy Place, Box 1230, New \\ York, NY 10029, USA \\ ${ }^{2}$ Fishberg Department of Neuroscience, Icahn School of Medicine at Mount \\ Sinai, New York, NY, USA \\ ${ }^{3}$ Friedman Brain Institute, Icahn School of Medicine at Mount Sinai, New York, \\ NY, USA
}

Published online: 29 June 2014

(C) Springer International Publishing AG 2014

Keywords Agoraphobia - Anxiety - Benzodiazepines (BZD) - Beta-blockers - Buspirone - Cognitive behavioral therapy (CBT) - D-cycloserine (DCS) - Exposure and response prevention (ERP) · Generalized anxiety disorder (GAD) - Monoamine oxidase inhibitor (MAOI) - Obsessive-compulsive disorder (OCD) • Panic disorder (PD) • Psychotherapy - Serotonin norepinephrine reuptake inhibitors (SNRI) - Serotonin selective reuptake inhibitors $($ SSRI) - Social anxiety disorder (SAD) - Specific phobia (SP) - Tricyclic antidepressant (TCAs)

\section{Opinion statement}

Anxiety disorders are prevalent and represent an important focus of treatment within the field of psychiatry as well as within medicine more broadly. First-line pharmacotherapy treatment for anxiety disorders is serotonin selective reuptake inhibitors (SSRIs) or serotonin norepinephrine reuptake inhibitors (SNRIs). For patients who do not responsd to an initial first-line treatment, clinicians should ensure that there has been adequate exposure to the medication by assessing compliance and optimizing the prescribed dose. Non-response to a treatment trial should also prompt a re-evaluation of the diagnosis and a search for occult psychiatric, substance, or general medical disorders. Laboratory tests and other components of a diagnostic work-up should be considered if they have not already been completed. Following confirmation of the diagnosis, the clinician should consider a switch to an agent from a different class, for example a tricyclic antidepressant or monoamine oxidase inhibitor. Combination treatments with an antidepressant plus a benzodiazepine, second-generation antipsychotic, anticonvulsant, $\beta$-blocker, or other medication may be considered but data is limited. Psychotherapy is an important treatment component for anxiety disorders and should be implemented whenever feasible. Variants of cognitive behavioral therapy (CBT) in particular are effective in reducing anxiety symptoms, and data suggest that the com- 
bination for CBT plus medication may be particularly beneficial for patients. Obsessivecompulsive disorder $(O C D)$, while sharing many clinical features with anxiety disorders, represents its own unique clinical challenge and has been removed from the category of anxiety disorders in the 5th edition of the Diagnostic and Statistical Manual of Mental Disorders (DSM-V). SSRIs are first-line therapy for OCD and higher doses are often required compared with anxiety disorders or major depressive disorder. Exposure and response prevention may be a particularly helpful form of psychotherapy for this patient population. For severe, intractable $0 C D$, deep brain stimulation may be an appropriate therapeutic option.

\section{Introduction}

Anxiety disorders are among the most prevalent psychiatric disorders [1] and account for a substantial public health burden. Obsessive-compulsive and related disorders, while not as common as anxiety disorders, are often associated with marked functional impairment and illness burden. However, therapeutic approaches to anxiety and obsessive-compulsive disorders (OCDs) are relatively understudied in comparison to other psychiatric disorders. The Diagnostic and Statistical Manual of Mental Disorders, 5th edition (DSM-V) [2] includes eight distinct anxiety disorders: generalized anxiety disorder (GAD), panic disorder (PD), agoraphobia, social anxiety disorder (SAD), specific phobia (SP), separation anxiety disorder, selective mutism and anxiety disorder due to another medical condition. Beginning with DSM-V, obsessive-compulsive and related disorders are classified as a distinct set of illnesses. Within this new scheme, obsessive-compulsive and related disorders include OCD, body dysmorphic disorder, hoarding disorder, trichotillomania, and excoriation disorder.

Anxiety disorders are commonly associated with functional impairment, maladaptive cognitive biases, excessive worry and physiological symptoms of anxiety or stress. This group of disorders is characterized by excessive fear responses, including autonomic arousal, muscle tension, vigilance, and avoidant behavior. Individual anxiety disorders differ in terms of the specific phenomenology and clinical presentation. GAD has a a lifetime prevalence of 5-6\%, and key symptoms are excessive worry and anxiety that is difficult to control, causing significant distress and impairment, and occurring on more days than not for at least 6 months [2]. Additionally, many patients experience somatic symptoms such as elevated blood pressure, increased heart rate, and muscle tension. PD is a chronic illness characterized by recurrent, unexpected panic attacks, accompanied by intense fear or discomfort due to attacks. Attacks occur where there is seemingly nothing threatening to the individual. The intense fear is typically accompanied by a set of cognitive and physical symptoms (i.e. depersonalization, heart palpitations, shortness of breath) and ongoing worry for at least 1 month about experiencing another attack. Of note, panic attacks can occur in the context of other anxiety disorders. Agoraphobia is marked by intense fear, anxiety, and avoidance of specific scenarios, such as using public transportation, being in open spaces, or standing in lines or crowds. Agoraphobia can also complicate PD and other anxiety disorders. Social phobia, or SAD, is a condition marked by anxiety in reaction to social or performance situations, often leading to avoidance behavior [2]. Comorbitity is common with SAD and often precedes the onset of other disorders such as major depressive disorder (MDD) and substance use disorders [3]. SP is similar to SAD, but anxiety is provoked by an explicit stimulus, such as animals or specific natural environments. Childhood anxiety disorders, such as selective mutism (consistent failure to speak in certain social situations) and separation anxiety disorder (developmentally inappropriate and excessive fear of being separated from attachment figures) will be discussed briefly in the pediatric considerations.

Obsessive-compulsive and related disorders are now classified together in DSM-V, in part due to similarities in clinical presentation, developmental course, and presumed shared underlying biological features. OCD is characterized by recurrent, intrusive, and distressing thoughts, images, or impulses, and repetitive mental or behavioral acts that the individual feels driven to perform to prevent or reduce distress [2]. For a discussion of other obsessive-compulsive and related disorders, the reader is referred to Charney et al. $[4 \bullet \bullet]$. 
In this article, current treatment options for anxiety disorders and OCD will be reviewed and future treatment directions will also be considered. Post-traumat- ic stress disorder (PTSD) and related disorders have been removed from the anxiety disorders class and will not be discussed in this article.

\section{Treatment}

\section{Pharmacologic treatment of anxiety disorders}

- Antidepressant medications are the most common agents used for the treatment of anxiety disorders. First-line treatment is typically serotonin selective reuptake inhibitors (SSRIs) because of their efficacy, tolerability, and general safety profile. Likewise, serotonin norepinephrine reuptake inhibitors (SNRIs) are commonly used in the treatment of anxiety disorders and have a favorable safety profile similar to SSRIs [5].

- Tricyclic antidepressants (TCAs) and monoamine oxidase inhibitors (MAOIs) are limited by safety and tolerability issues but should be considered in treatment-resistant patients [6].

- If the initial treatment trial is either partially effective or ineffective, next-step options include dose optimization, augmentation, combination, or switching agents.

- Benzodiazepines are commonly utilized as adjunct treatment for anxiety disorders [6].

- Consuming alcohol or illicit substances may decrease the benefits and increase the adverse effects of pharmacologic agents, particularly for benzodiazepines and buspirone.

Selective serotonin reuptake inhibitors and serotonin norepinephrine reuptake inhibitors

Several randomized controlled trials (RCTs) support drugs in the SSRI and SNRI classes as the first-line therapeutic option for GAD, SAD, PD, and $\mathrm{OCD}[3,7,8]$. For treatment considerations, specific agents are often chosen for their side effect profile on a case-by-case basis. US FDA approvals to treat specific anxiety disorders include paroxetine (GAD, OCD, PD and SP), sertraline (OCD, PD, SP), citalopram, escitalopram (GAD), fluoxetine (OCD, PD), fluvoxamine (OCD, SP), venlafaxine (GAD, PD, $\mathrm{SP})$, and duloxetine (GAD).

Standard dosage

Varies by agent (see Table 1). To minimize side effects, starting with the lowest minimal effective dose and slowly increasing the dose as needed is recommended. Dose adjustments are made according to patient response, tolerability, and clinical urgency.

Contraindications

Such agents should be used with caution in combination with other serotonergic medications that may cause serotonin syndrome. In particular, the concomitant use of SSRIs or SNRIs with a MAOI is contraindicated. Duloxetine is contraindicated in patients with uncontrolled narrow-angle glaucoma. 


\begin{tabular}{|c|c|c|c|}
\hline Pharmacologic class & Examples & US FDA-approved indications & Dose range (mg daily) \\
\hline \multirow[t]{4}{*}{ SSRI } & Escitalopram & GAD & $10-20$ \\
\hline & Fluoxetine & $O C D, P D$ & $20-60$ \\
\hline & Fluvoxamine & OCD, SP & $100-300$ \\
\hline & Paroxetine & $\mathrm{GAD}, \mathrm{OCD}, \mathrm{PD}, \mathrm{SP}$ & $20-50$ \\
\hline \multirow[t]{2}{*}{ SNRI } & $\begin{array}{l}\text { Sertraline } \\
\text { Duloxetine }\end{array}$ & $\begin{array}{l}\text { OCD, PD, SP } \\
\text { GAD }\end{array}$ & $\begin{array}{l}50-200 \\
60-120\end{array}$ \\
\hline & Venlafaxine & GAD, PD, SP & $75-225$ \\
\hline \multirow[t]{6}{*}{ BZD } & Alprazolam & Anxiety (non-specific), PD & $1-4$ \\
\hline & Chlordiazepoxide & Anxiety (non-specific) & $15-40$ \\
\hline & Clonazepam & PD & $1-4$ \\
\hline & Diazepam & Anxiety (non-specific) & $2-10$ \\
\hline & Lorazepam & Anxiety (non-specific) & $1-6$ \\
\hline & 0xazepam & Anxiety (non-specific) & $30-120$ \\
\hline \multirow[t]{3}{*}{ TCA } & Clomipramine & $O C D, P D$ & $25-250$ \\
\hline & Doxepine & Anxiety (non-specific) & $75-300$ \\
\hline & Imipramine & PD & $100-200$ \\
\hline MAOI & Phenelzine & PD & $45-90$ \\
\hline Antihistamine & Hydroxyzine & Anxiety (non-specific) & $200-400$ \\
\hline Other & Buspirone & Anxiety (non-specific) & $20-60$ \\
\hline
\end{tabular}

Main drug interactions Must avoid simultaneous treatment with MAOIs. Citalopram, escitalopram, and sertraline have relatively few drug-drug interactions. SSRIs and duloxetine are inhibitors of the hepatic cytochrome P450 (CYP) enzyme CYP2D6 [6].

Main side effects Common side effects include nausea, drowsiness, sexual dysfunction, somnolence, weight gain or loss, insomnia, anxiety, dizziness, headache, restlessness, agitation, diarrhea, and dry mouth. Discontinuation syndrome is common with venlafaxine. Citalopram may extend the QT interval and caution should be exercised when used in patients with cardiac conditions [9].

Special points Transient nervousness, jitteriness or worsening of anxiety at the onset of treatment with an SSRI may occur [9]. Adjunctive treatment with a benzodiazepine at the start of treatment with an SSRI and SNRI can mitigate this increase in anxiety. Another limitation commonly observed with monoaminergic treatments is the delay in onset of therapeutic effect of several weeks [6].

Cost Many of the SSRIs are available in generic form, including fluoxetine, paroxetine, sertraline, citalopram, escitralopram, and fluvoxamine. The SNRI venlafaxine is also available in generic formulation. 
Standard dosage

Contraindications

Main drug interactions

Main side effects

Special points
TCAs have been shown to be efficacious in the treatment of patients with GAD, PD, and OCD. They are considered second-line agents for the treatment of anxiety disorders, primarily due to their side effect burden [10]. Despite the potential for side effects, these medications remain an important treatment option for patients who do not respond to first-line treatment. Imipramine is FDA approved for the treatment of GAD and $\mathrm{PD}$, while clomipramine is approved for the treatment of OCD and PD.

Doses vary (see Table 1). Start at a low dose in order to avoid side effects, and slowly increase the dose. TCAs vary in their response depending on tolerability, side effects, body mass index, and how rapidly the drug is metabolized [11]. Patients should be slowly tapered off the medication in order to prevent withdrawal symptoms.

TCAs are contraindicated in patients who have narrow-angle glaucoma, liver disease, enlarged prostate, heart conditions, or a history of seizures [12].

TCAs are metabolized via CYP. TCAs should not be combined with MAOIs [11].

Side effects include dry mouth, constipation, urinary retention, somnolence, dizziness, weight gain, sexual dysfunction, and orthostasis. There is an increased risk of cardiotoxicity in overdose and TCAs have less-acceptable tolerability profiles compared with SSRIs. Toxicity may be due to QT interval prolongation and may lead to arrhythmias, and could be fatal in overdose [11].

Due to their relative side effect burden, TCAs are generally reserved for second- or third-line treatment trials. However, they remain an important treatment option for some patients.
Standard dosage

Contraindications

Main drug interactions

Main side effects

Special points
Similar to TCAs, MAOIs are not usually the first pharmacological option for anxiety disorder due to a greater potential side effect burden. Given the risk of serious adverse events and dietary restrictions associated with MAOIs, they are generally reserved as second- or third-line agents or for refractory SAD or PD [13]. Phenelzine is approved for the treatment of SAD and PD, and represents an important third- or fourthline agent for refractory patients [14, 15].

Doses vary (see Table 1).

MAOIs can cause dangerous interactions with certain foods, such as some cheeses, broad beans, pickled foods, and foods containing high levels of tyramine. Beverages to avoid include beer and red wine [16].

MAOIs have major interactions with many other drugs, including anesthetics, analgesics, other antidepressants, and anxiolytics.

Up to $50 \%$ of patients experience dizziness; other common side effects include dry mouth, headache, fatigue, weight gain, sexual dysfunction, somnolence, gastrointestinal problems, and urinary problems [16].

MAOIs are considered top treatment in cases of anxiety or depression with atypical features (i.e. a heavy feeling in the arms and legs, sensitivity to rejection, and a reactive mood). 
Standard dosage Doses vary depending on the potency of the particular benzodiazepine (see Table 1). Benzodiazepines are generally started at a low dose and are titrated up as needed based on response.

Contraindications Benzodiazepines should be used with caution in patients with myasthenia gravis, sleep apnea, bronchitis, and chronic obstructive pulmonary disease (COPD) [18].

Main drug interactions Benzodiazepines may lead to elevated methadone peak levels and other drug interactions via common CYP3A4 oxidation pathways in the liver.

Main side effects These include impairment of psychomotor performance, somnolence, appetite change, fatigue, dependence, and withdrawal symptoms after longterm treatment, and rebound anxiety after short-term treatment [18].

Special points The use of benzodiazepines may place the patient at risk for developing tolerance or dependence, and vigilance should be used when prescribing these medications.

Serotonergic agonist - buspirone

Standard dosage

Contraindications

Main drug interactions

Main side effects

Special points
Buspirone, a serotonergic azapirone agent, is FDA approved as a treatment for GAD [19]. The pharmacological profile of buspirone is unique in its function as a selective agonist of the $5-\mathrm{HT}_{1 \mathrm{~A}}$ receptor. Despite reasonable evidence for its efficacy, buspirone is not generally considered first-line treatment for GAD due to its slow onset of action and tolerability issues at higher doses [20].

Doses range from $20-60 \mathrm{mg}$ daily.

For patients with hypersensitivity to buspirone or any component of the formulation, the drug is contradicted. Buspirone should not be used in patients who have metabolic acidosis, as in diabetes and severely compromised liver and or renal function.

Buspirone may interact with MAOIs and cause increased blood pressure. The combination of buspirone and trazodone may cause abnormal liver enzymes in the blood. The concurrent use of grapefruit juice should be avoided.

Dizziness, nausea, headache, nervousness, lightheadedness, excitement, and insomnia.

Advantages of buspirone include low potential for cognitive or motor impairment and absence of abuse liability.

Other pharmacological approaches
Pregabalin is an anticonvulsant drug currently approved as an adjunctive treatment for seizure disorder, and has shown efficacy for GAD com- 
pared with placebo in several RCTs, although it never received FDA approval for this indication $[21,22]$.

Standard dosage Doses range from 50-300 mg.

Contraindications Hypersensitivity to pregabalin or any of its components.

Main drug interactions Alcohol and drugs that cause sedation may increase the sedative effects of pregabalin [22].

Main side effects Dizziness, drowsiness, dry mouth, edema, blurred vision, weight gain, and difficulty concentrating [22].

Special points Pregabalin is not approved for treating GAD by the FDA; however, it has recently been approved for the treatment of anxiety in Europe [22].

\section{$\beta$-blockers}

\begin{abstract}
Standard dosage A dose of 20-60 mg may be given 30-60 min before an anxiety-inducing situation.
\end{abstract}

Contraindications Patients who have a history of $\beta$-blocker intolerance or allergy, asthma, diabetes, certain cardiac conditions, and chronic lung disease.

Main side effects Side effects are rare; however, some side effects may include lightheadedness, sleepiness, problems with short-term memory, unusually slow pulse, lethargy, insomnia, diarrhea, cold hands and feet, numbness and/or tingling of fingers and toes [6].

Special points $\beta$-blockers, while helpful, may not provide complete symptom relief; however, in contrast to benzodiazepines, they are devoid of abuse liability.

\section{Pharmacologic treatment of obsessive-compulsive disorder}

While the etiology and pathophysiology of OCD are not completely understood, evidence-based treatments including pharmacotherapy with

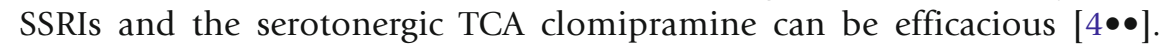
Clomipramine can lead to improvement in 40-60\% of people with OCD [24]; however, it is recommended that patients first begin treatment with an SSRI because they have a better side effect profile for OCD and can be effective.

Fluoxetine, fluvoxamine, sertraline, and paroxetine have all been FDAapproved for the treatment of OCD, as well as the TCA clomipramine.

Standard dosage SSRIs at the high end of the dosing range may be necessary in the treatment of OCD. It is advised that patients start at a low dose for tolerability and that the dose be increased to the highest maximum dose before deciding that a patient is resistant. To note, the dosing levels of the TCA clomipramine are typically higher than SSRIs (i.e. 25-500 mg). 


\begin{abstract}
Main side effects In addition to the side effects for SSRIs, clomipramine can cause dry mouth, constipation, delayed urination, sedation, weight gain, orthostatic hypotension, and cardiac conduction abnormalities or seizures.

Special points As these treatments may only be effective in relieving symptoms for $50 \%$ of patients, and relapse can occur if the medication is discontinued, one strategy is to switch to another SSRI or eventually clomipramine.
\end{abstract}

\title{
Neurostimulation and surgical options of $O C D$
}

The following neurostimulation and surgical options may be utilized in the case of treatment-refractory anxiety disorders, including OCD. For example, subsets of OCD patients (10\%) do not respond to the pharmacologic and psychotherapy strategies. These treatment-resistant patients are candidates for neurosurgical intervention.

- Deep brain stimulation (DBS): DBS is a neurosurgical procedure that implants a brain pacemaker to send electrical impulses to specific parts of the brain. Research has indicated DBS as a safe treatment for movement disorders (i.e. Parkinson's disease) and recent trials have focused on its therapeutic potential for depression and OCD. DBS is reserved for treatment-refractory and severely debilitating OCD [25].

- Repetitive transcranial magnetic stimulation (rTMS): rTMS is an upcoming and non-invasive therapy option for OCD that produces fluctuating magnetic pulses through an electric current passing through a metal coil applied on the patient's scalp. Early research has indicated that rTMS may help alleviate symptoms for OCD patients [26, 27].

- Electroconvulsive therapy (ECT): Several case reports suggest that ECT may be effective for severe OCD, particularly for OCD with comorbid depressive symptoms [28].

\section{Psychotherapeutic treatment of anxiety disorder}

For the treatment of many anxiety disorders, psychotherapy is a first-line option. Psychotherapy aims to explore the root of worries and fears and develop enhanced coping and problem-solving skills. Since each anxiety disorder differs in its symptom profile and presumed etiology, therapies are targeted to a specific disorder or presentation. The leading therapeutic approaches in the treatment of anxiety disorders are cognitive behavioral therapy (CBT) and exposure therapy; other forms of psychotherapy will also be reviewed. For OCD, the gold-standard psychotherapy is exposure and response prevention (ERP).

For many patients, the combination of psychotherapy with an SSRI or other medication can be particularly helpful. For example, the combination of CBT and benzodiazepines [29] or sertraline [30] may be more effective in treating GAD than medications alone.

CBT for anxiety disorders aims to address cognitive symptoms (i.e. excessive and uncontrollable worry), physical symptoms (i.e. motor tension, vigilance, restlessness) and behavior symptoms (i.e. excessive 
preparation, procrastination, avoidance) $[31 \bullet \bullet, 32]$. Six primary techniques are often utilized in the treatment of various anxiety disorders. The frequency and duration of CBT sessions differs based on the disorder and symptom severity. Research also supports using Internet or computerized CBT programs for GAD. Internet CBT (iCBT) programs use online lessons with printable summaries, homework assignments, and online resources to manage sleep problems, low mood, and comorbid symptoms [33].

- Patient psychoeducation: The patient and therapist review a treatment plan and the rationale for the proposed approach [33]. Psychoeducation focuses on misconceptions regarding anxiety and is targeted to the primary disorder (i.e. in PD, explanations of the fight-fight response and the physiological symptoms that occur during panic and anxiety) [34].

- Self-monitoring: Introduced in the first session and continued throughout the course of treatment, patients are encouraged to be 'personal scientists' who observe their reactions and keep records. For GAD, patients use a 'Worry Record' to chart their progress, while patients with PD record triggers for panic attacks [34].

- Relaxation training: Progressive muscle relaxation techniques are used to assist with raised muscle tension and lowered flexibility of autonomic functioning $[31 \bullet \bullet]$. Patients briefly and deliberately tense muscle groups in a systematic manner (16 muscle groups, then 8 , then 4). Breathing retraining teaches slow, abdominal breathing exercises [35] and may be particularly helpful for PD.

- Cognitive restructuring: Therapists help patients identify common misconceptions in thinking (i.e. overestimating the probability of a negative consequence) as well as rigid rules or beliefs. Using an empirical approach, patients examine the validity of their thoughts to re-interpret situations $[31 \bullet \bullet]$.

- Exposure: Exposure techniques vary greatly between the different anxiety disorders.

- Imaginal exposure: Specified to tolerate negative affect and auto-

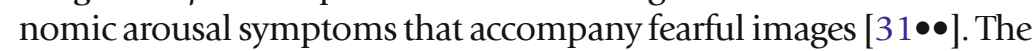
therapist guides the patient through systematic exposure to images, moving up a fear hierarchy once anxiety is reduced to a mild level.

- In vivo exposure: Considered the gold-standard of exposure therapy, in vivo is mostly utilized in the treatment of SP and PD. Patients confront the feared stimulus in a safe and controlled manner [36]. However, this method is emotionally taxing and the refusal rate can range between 14 and $27 \%$ [37].

- Virtual reality exposure (VRE): VRE, designed mainly for the treatment of SP, allows the patient to experience the phobia with computer stimulation. VRE is used for phobias that are expensive to reproduce (e.g. flying) [38, 39].

- Social skills training: Unique to treatment of SAD, social skills training works to remedy behavioral deficiencies (i.e. poor eye contact). Social 
skills training incorporates techniques such as therapist modeling, corrective video feedback, and behavioral rehearsal [39].

- Acceptance and commitment therapy (ACT) uses mindfulness strategies focused on acceptance of internal states and orienting actions toward valued goals [40].

- In contrast to CBT, ACT does not utilize cognitive restructuring or any attempts to correct somatic dysregulation (i.e. progressive muscle relaxation) [41]. Acceptance-based approaches have been most researched in the treatment of GAD, SAD, and PD.

- More frequently studied for the treatment of SAD, attention retraining modifies attention bias through guided attention to certain stimuli with dot-probe detection tasks [42].

- In a dot-probe task, the patient sees two stimuli simultaneously appear on a computer screen. One stimulus is neutral while the other is threatening; for instance, two pictures of the same person would appear, with one photograph depicting an emotional expression and the other a neutral disposition. Stimuli are then removed and replaced with a probe (dot or a line) [42].

- For attention retraining, the connection between the probe and non-threatening stimulus is strengthened and others are weakened [42].

- Interpersonal psychotherapy (IPT) aims to address interpersonal difficulties leading to psychological problems [43], and differs from CBT in several key areas. IPT is less structured and focuses on feelings in interpersonal situations rather than cognitions [43].

- The main premise of IPT is that symptoms reflect a treatable illness and should not be seen as a fault of the patient. Second, IPT is grounded in the belief that symptoms arise in an interpersonal context [43].

\section{Psychotherapeutic treatment of OCD}

CBT is the first-line treatment for OCD; however, the techniques differ somewhat compared with procedures for other anxiety disorders. Similar to CBT for most anxiety disorders, patients with OCD begin with treatment planning and psychoeducation. In particular, the therapist introduces the patient to the notion of response prevention procedures and encourages self-monitoring of typical rituals. Specific to OCD is ERP therapy. 
- ERP is similar to traditional exposure therapy, except that special attention is paid to the prevention of escape responses.

- Therapy employs in vivo and imaginal exposure and is most efficacious when patients remain in the exposure situation until the compulsive rituals and the obsessional distress reduce spontaneously [44, 45].

- Cognitive therapy (CT) techniques can augment ERP by assisting patients to confront maladaptive thought patterns or mistaken beliefs. Specific CT techniques include the 'cognitive continuum technique', where patients rate their level of immorality for any unacceptable obsessive intrusive thoughts (i.e. blasphemy, violent thoughts) while also rating the morality of persons who have committed violent acts. Patients then re-rate themselves and re-evaluate their personal level of morality for only having intrusive thoughts [44, 46].

Previously, the focus of pharmacological research in anxiety disorders was on the role of the monoamine system. Now there has been a shift in experimental therapeutics towards novel mechanisms, such as the endocannabinoid system, the glutamate system, and neuropeptides that regulate stress and fear reactions $[47 \bullet \bullet]$.

- The glutamate system has been implicated as a relevant mechanism in the central nervous system for mood and anxiety disorders. Ketamine, a glutamate N-methyl-d-aspartate (NMDA) receptor antagonist, has shown rapid antidepressant effects after a single administration in treatment-resistant MDD (TRD) [48].

Ketamine is also being tested as a treatment for anxiety. Preliminary evidence from recent proof-of-concept RCTs have demonstrated rapid reduction in anxiety symptoms in stress-related psychiatric disorders, including for the treatment of OCD [49], as well as PTSD [50].

- Riluzole, also a glutamate modulator, is approved for the treatment of amyotrophic lateral sclerosis (ALS). Riluzole is currently being studied as a potential adjunctive agent in TRD, with ongoing trials in

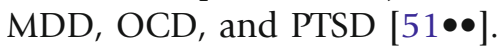

- A new promising approach is augmenting the effects of CBT with the partial NMDA receptor agonist D-cycloserine for the treatment of

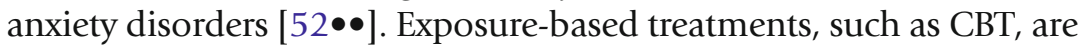
dependent on extinction strategies to treat the core fears underlying the anxiety disorder. Based on the understanding that fear learning and extinction are influenced by the NMDA receptor, D-cycloserine is now being studied in human trials to augment such extinction strategies. Promising initial results have been reported for phobias,

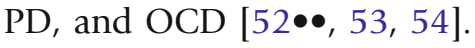




\section{Pediatric considerations}

- The neuropeptide Y (NPY) system is a promising emerging target for novel anxiety therapeutics. NPY and its receptors demonstrate significant levels in brain regions that are implicated in anxiety, such as the amygdala and the hippocampus [55]. Lower levels of NPY are associated with an increase in fear, anxiety, and reduced stress resilience. Therefore, therapeutic strategies focusing on increasing NPY signaling in the brain may represent a promising avenue for future research.

- Vilazodone is a combined SSRI and partial 5- $\mathrm{HT}_{1 \mathrm{~A}}$ receptor agonist currently FDA-approved for MDD. Vilazodone is currently in latephase testing for GAD and SAD [51]

- Agomelatine is a melatonin receptor agonist that has been the focus of research and clinical attention in Europe as an antidepressant but has also demonstrated efficacy in a phase II trial in GAD [51]. It is not currently approved in the US.

- Anxiety disorders unique to children are separation anxiety disorder and selective mutism. For anxiety disorders, only CBT, with and without family anxiety management, is shown to be efficacious. For childhood phobias, imaginal exposure, in vivo exposure, filmed modeling, and cognitive behavioral interventions are the most wellestablished treatment options [56].

\section{Compliance with Ethics Guidelines}

\section{Conflict of Interest}

Sehrish Sayed declares that she has no conflict of interest.

Sarah R. Horn declares that she has no conflict of interest.

In the past two years, Dr. Murrough has received research support from the National Institutes of Health, the National Institute of Mental Health, the Department of Veterans Affairs, the Doris Duke Charitable Foundation, the American Foundation for Suicide Prevention, the Brain and Behavior Research Foundation, Janssen Research and Development and Avanir Pharmaceuticals; he has served on advisory boards for Janssen Research and Development and Genentech and has provided consultation services for ProPhase, LLC and Impel Neuropharma. Dr. Murrough is named on a filed patent application for neuropeptide Y in the treatment of mood and anxiety disorders. Institutional conflict: Dr. Charney (Dean of Icahn School of Medicine at Mount Sinai), and Icahn School of Medicine at Mount Sinai have been named on use patents on ketamine for the treatment of depression and on ketamine for the treatment of PTSD. If ketamine were shown to be effective in the treatment of depression or PTSD, Dr. Charney and Icahn School of Medicine at Mount Sinai could benefit financially.

Human and Animal Rights and Informed Consent

This article does not contain any studies with human or animal subjects performed by any of the authors. 


\section{References and Recommended Reading}

Papers of particular interest, published recently, have been highlighted as:

$\bullet \quad$ Of major importance

1. Kessler RC, Berglund P, Demler O, Jin R, Merikangas KR, Walters EE. Lifetime prevalence and age-of-onset distributions of DSM-IV disorders in the National Comorbidity Survey Replication. Arch Gen Psychiatry. 2005;62:593-602.

2. American Psychiatric Association. Diagnostic and statistical manual of mental disorders. 5th ed. Washington, DC: American Psychiatric Association; 2013.

3. Stein $M B$, Stein DJ. Social anxiety disorder. Lancet. 2008;371:1115-25.

4.• Charney DS, Nestler EJ, Sklar P, Buxbaum JD. Neurobiology of mental illness. Oxford: Oxford University Press; 2013.

This textbook is the most recent edition of the Neurobiology of mental illness series. Chapters 39-49 detail the etiology, presentation, and treatment of anxiety and obsessive-related disorders (Class IV).

5. Bandelow B, Sher L, Bunevicius R, Hollander E, Kasper S, Zohar J, et al. Guidelines for the pharmacological treatment of anxiety disorders, obsessive-compulsive disorder and posttraumatic stress disorder in primary care. Int J Psychiatry Clin Pract. 2012;16:77-84.

6. Ravindran LN, Stein MB. The pharmacologic treatment of anxiety disorders: a review of progress. J Clin Psychiatry. 2010;71:839-54.

7. Baldwin DS, Waldman S, Allgulander C. Evidencebased pharmacological treatment of generalized anxiety disorder. Int J Neuropsychopharmacol. 2011;14:697-710.

8. Beekman A, Cuijpers P, Reynolds C, Sijbrandij E, Koole S, Andersson G. The efficacy of psychotherapy and pharmacotherapy in treating depressive and anxiety disorders: a meta-analysis of direct comparisons. World Psychiatry. 2013;12(2):137-48.

9. Brambilla P, Cipriani A, Hotopf M, Barbui C. Sideeffect profile of fluoxetine in comparison with other SSRIs, tricyclic and newer antidepressants: a metaanalysis of clinical trial data. Pharmacopsychiatry. 2005;38(2):69-77.

10. Bakker A, Van Balkom A, Spinhoven P. SSRIs vs. TCAs in the treatment of panic disorder: a metaanalysis. Acta Psychiatr Scand. 2002;106:163-7.

11. Keller MB. The long-term clinical course of generalized anxiety disorder. J Clin Psychiatry. 2002;63 Suppl 8:11-6.

12. Kapczinski F, Lima M, Souza J, Schmitt R. Antidepressants for generalized anxiety disorder. Cochrane Database Syst Rev. 2003;2, CD003592.
13. Lanouette NM, Stein MB. Advances in the management of treatment-resistant anxiety disorders. FOCUS J Lifelong Learn Psychiatry. 2010;8:501-24.

14. Blanco C, Heimberg RG, Schneier FR, Fresco DM, Chen H, Turk CL, et al. A placebo-controlled trial of phenelzine, cognitive behavioral group therapy, and their combination for social anxiety disorder. Arch Gen Psychiatry. 2010;67:286-95.

15. Batelaan NM, Van Balkom AJ, Stein DJ. Evidencebased pharmacotherapy of panic disorder: an update. Int J Neuropsychopharmacol. 2012;15:403-15.

16. Virani AS, Bezchlibnyk-Butler KZ, Jeffries JJ, Procyshyn RM. Clinical handbook of psychotropic drugs. Hogrefe; 2012.

17. McHugh RK, Smits JA, Otto MW. Empirically supported treatments for panic disorder. Psychiatr Clin N Am. 2009;32:593-610.

18. Allgulander C, Msghina M. Benefits and risks of benzodiazepines: a current analysis of facts. Lakartidningen. 2011;108:2025-9.

19. Chessick C, Allen M, Thase M, Batista Miralha da Cunha AB, Kapczinski F, de Lima M, et al. Azapirones for generalized anxiety disorder. Cochrane Database Syst Rev. 2006;(3):CD006115.

20. Davidson JR, DuPont RL, Hedges D, Haskins JT. Efficacy, safety, and tolerability of venlafaxine extended release and buspirone in outpatients with generalized anxiety disorder. J Clin Psychiatry. 1999;60(8):528-35.

21. Montgomery SA, Tobias K, Zornberg GL, Kasper S, Pande AC. Efficacy and safety of pregabalin in the treatment of generalized anxiety disorder: a 6-week, multicenter, randomized, double-blind, placebocontrolled comparison of pregabalin and venlafaxine. J Clin Psychiatry. 2006;67:771-82.

22. Lydiard RB, Rickels $K$, Herman B, Feltner DE. Comparative efficacy of pregabalin and benzodiazepines in treating the psychic and somatic symptoms of generalized anxiety disorder. Int J Neuropsychopharmacol. 2010;13:229-41.

23. Bourgeois $M$, James A. The management of performance anxiety with beta-adrenergic blocking agents. Jefferson J Psychiatry. 2011;9:5.

24. Simpson HB. Pharmacological treatment of obsessive-compulsive disorder. In: Stein MB, Steckler $\mathrm{T}$, editors. Behavioral neurobiology of anxiety and its treatment. Springer; 2010: pp. 527-43. 
25. Morishita T, Fayad SM, Goodman WK, Foote KD, Chen D, Peace DA, et al. Surgical neuroanatomy and programming in deep brain stimulation for obsessive compulsive disorder. Neuromodulation. Epub 17 Dec 2013.

26. Pogarell O, Juckel G, Mavrogiorgou P, Mulert C, Folkerts M, Hauke W, et al. Symptom-specific EEG power correlations in patients with obsessive-compulsive disorder. Int J Psychophysiol. 2006;62:87-92.

27. Margoob MA, Chandel R, Mushtaq H, Mushtaq D. Treatment-resistant obsessive-compulsive disorder (OCD): current understanding. In: Kalin V, editor. Obsessive-compulsive disorder: the old and the new problems. Croatia: Intech; 2014.

28. Liu X, Cui H, Wei Q, Wang Y, Wang K, Wang C, et al. Electroconvulsive therapy on severe obsessive-compulsive disorder comorbid depressive symptoms. Psychiatry Investig. 2014;11:210-3.

29. Power KG, Simpson R, Swanson V, Wallace L, Feistner A, Sharp D. A controlled comparison of cognitive-behaviour therapy, diazepam, and placebo, alone and in combination, for the treatment of generalised anxiety disorder. J Anxiety Disord. 1990;4:267-92.

30. Walkup JT, Albano AM, Piacentini J, Birmaher B, Compton SN, Sherrill JT, et al. Cognitive behavioral therapy, sertraline, or a combination in childhood anxiety. N Engl J Med. 2008;359:2753-66.

31.• Wells A. Cognitive therapy of anxiety disorders: a practice manual and conceptual guide. John Wiley \& Sons; 2013.

This manual is a comprehensive and detailed guide to cognitive therapy techniques often utilized in the treatment of anxiety disorders (Class IV).

32. Hoge EA, Ivkovic A, Fricchione GL. Generalized anxiety disorder: diagnosis and treatment. BMJ. 2012;345:e7500.

33. Robinson E, Titov N, Andrews G, McIntyre K, Schwencke G, Solley K. Internet treatment for generalized anxiety disorder: a randomized controlled trial comparing clinician vs technician assistance. PloS One. 2010;5:e10942.

34. Clark DM. A cognitive approach to panic. Behav Res Ther. 1986;24:461-70.

35. Taylor S. Understanding and treating panic disorder: cognitive-behavioural approaches. John Wiley \& Sons Ltd; 2000.

36. Wolitzky-Taylor KB, Horowitz JD, Powers MB, Telch MJ. Psychological approaches in the treatment of specific phobias: a meta-analysis. Clin Psychol Rev. 2008;28:1021-37.

37. Antony MM, Swinson RP. Phobic disorders and panic in adults: a guide to assessment and treatment. American Psychological Association; 2000.

38. Rothbaum BO. Using virtual reality to help our patients in the real world. Depress Anxiety. 2009;26(3):209-11.
39. Herbert JD, Gaudiano BA, Rheingold AA, Myers VH, Dalrymple K, Nolan EM. Social skills training augments the effectiveness of cognitive behavioral group therapy for social anxiety disorder. Behav Ther. 2005;36:125-38.

40. Hayes SC, Strosahl KD, Wilson KG. Acceptance and commitment therapy: an experiential approach to behavior change. Guilford Press; 1999.

41. Arch JJ, Craske MG. Acceptance and commitment therapy and cognitive behavioral therapy for anxiety disorders: different treatments, similar mechanisms? Clin Psychol Sci Pract. 2008;15:263-79.

42. MacLeod C, Rutherford E, Campbell L, Ebsworthy G, Holker L. Selective attention and emotional vulnerability: assessing the causal basis of their association through the experimental manipulation of attentional bias. J Abnorm Psychol. 2002;111:107.

43. Markowitz JC, Lipsitz J, Milrod BL. Critical review of outcome research on interpersonal psychotherapy for anxiety disorders. Depress Anxiety. 2014;31(4):316-25.

44. Arumugham SS, Reddy YJ. Commonly asked questions in the treatment of obsessive-compulsive disorder. Expert Rev Neurother. 2014;14(2):151-63.

45. Lack CW. Obsessive-compulsive disorder: evidencebased treatments and future directions for research. World J Psychiatry. 2012;2(6):86-90.

46. Leahy RL. Cognitive therapy techniques: a practitioner's guide: Guilford Press; 2003.

47.• Griebel G, Holmes A. 50 years of hurdles and hope in anxiolytic drug discovery. Nat Rev Drug Discov. 2013;12:667-87.

This article is a comprehensive review of major trends from preclinical data within the past 50 years, as well as novel pharmacological treatment options for anxiety disorders (Class II).

48. Murrough JW, Perez AM, Pillemer S, Stern J, Parides $\mathrm{MK}$, Collins KA, et al. Rapid and longer-term antidepressant effects of repeated ketamine infusions in treatment-resistant major depression. Biol Psychiatry. 2013;74:250-6.

49. Rodriguez CI, Kegeles LS, Levinson A, Feng T, Marcus SM, Vermes D, et al. Randomized controlled crossover trial of ketamine in obsessive-compulsive disorder: proof-of-concept. Neuropsychopharmacology. 2013;38:2475-83.

50. Feder A, Parides MK, Murrough JW, Perez AM, Morgan JE, Saxena S, et al. Efficacy of intravenous ketamine for treatment of chronic posttraumatic stress disorder: a randomized clinical trial. JAMA Psychiatry. 2014;71(6):681-8.

51.• Garakani A, Murrough JW, Iosifescu DV. Advances in psychopharmacology for anxiety disorders. FOCUS J Lifelong Learn Psychiatry. 2014;12:152-62.

This paper presents novel treatment options from medication classes widely used in the treatment of anxiety disorders as well as novel mechanisms that are being targeted for emerging therapies (Class IV). 
52.•• Hofmann SG, Smits JA, Rosenfield D, Simon N, Otto MW, Meuret AE, et al. d-Cycloserine as an augmentation strategy with cognitive-behavioral therapy for social anxiety disorder. Am J Psychiatry. 2013;170:751-8.

This article describes recent findings on d-Cycloserine as an augmentative agent for CBT in SAD (Class I).

53. Otto MW, Tolin DF, Simon NM, Pearlson GD,

Basden S, Meunier SA, et al. Efficacy of d-cycloserine for enhancing response to cognitive-behavior therapy for panic disorder. Biol Psychiatry. 2010;67:365-70.
54. Bontempo A, Panza KE, Bloch MH. D-cycloserine augmentation of behavioral therapy for the treatment of anxiety disorders: a meta-analysis. J Clin Psychiatry. 2012;73:533-7.

55. Wu G, Feder A, Wegener G, Bailey C, Saxena S, Charney D, et al. Central functions of neuropeptide $\mathrm{Y}$ in mood and anxiety disorders. Expert Opin Ther Targets. 2011;15:1317-31.

56. Kendall PC. Child and adolescent therapy: cognitivebehavioral procedures. Guilford Press; 2011. 